\title{
IMPLEMENTATION OF UPFC MODEL INTO FAST DECOUPLED LOAD FLOW
}

\author{
Hazlie Mokhlis and Khalid Mohamed Nor \\ Department of Electrical Engineering, University of Malaya
}

\begin{abstract}
This paper describes the implementation of Unified Power Flow Controller (UPFC) steady-state model into Fast Decoupled load flow analysis. The model is integrated through sequential approach, where equations for solving these devices are separated from the basic Fast Decoupled equations. By using this approach, the basic Fast decoupled algorithm can be added with functionality to analyze UPFC without the need to modify the whole algorithm, which will require minimum modification. The proposed Fast Decoupled load flow has been tested using IEEE data test and shows it effectiveness in solving network containing single or multiple UPFC devices.
\end{abstract}

\section{INTRODUCTION}

Load flow analysis is one of the most important analysis in power system studies. Basically, it is used to determine voltage and phase angle of each bus-bar in the power system network, which are required for planning, operation and other purposes. The introduction of UPFC has made the basic load flow algorithm need to be enhanced to analyze the impact of UPFC in the power system network. This require a UPFC steady-state model for load flow application.

Many UPFC steady-state model has been proposed for load flow analysis $[2,3,4]$. Among these model, the steady-state model developed by C.R. Fuerter-Esquivel et. al. can be considered as the most flexible and effective model compared to the others [4]. The model was incorporated into Newton Raphson method (NRM) as the load flow solution method. By using this model, the load flow analysis has the ability to control active and reactive powers and voltage magnitude simultaneously, any one of them or any combination of them. These abilities are closely match to a practical UPFC application in power system network. On the other hand, other models have its own limitation such as can only work to control simultaneously all the parameters and cannot work to control selectively the parameters [2].

Although the steady-state model proposed by C.R. Fuerter-Esquivel et. al is flexible and effective, the implementation of the model into NRM algorithm is quite difficult. A few modifications need to be done to the basic algorithm. For example, the Jacobian matrix needs to be extended to include the differential equations of the UPFC steady-state model. This will involve of modifying many part mainly the codes of the NRM program if it has been previously developed. The NRM algorithm itself basically is also quite hard to be coded, it requires large memory and the computational process consumes a lot of time. Apart from those weaknesses, not all type of cases can be solved by using NRM. There are cases where this iterative analysis cannot meet convergence criteria such as if the initial value is chosen far from the exact correct value.

Convergence problem usually can be solved by using another well-known method i.e Fast Decoupled method (FDM). Some problems, which cannot be solved by using NRM may have solution by using FDM, although it may consumes a large number of iterations. Due to this capability and other FDM advantageous such as it provides high calculation speed, low memories storage requirement, high reliability and simplicity, we integrated this model into FDM. This study is also conducted since there are no studies that attempt to implement the UPFC model into FDM.

\section{UPFC STEADY STATE MODEL}

The UPFC steady-state model used in this study is shown in Fig. 1 [5]. UPFC basically consists of two voltage source back-to-back converters. A series converter is connected through a series transformer, and a shunt converter is connected through shunt transformer. These converters are operated from a common dc link provided by a dc storage capacitor. The role of the series converter is to inject a controllable voltage with the voltage magnitude, $V_{\text {sssc }}$ and phase angle $\theta_{\text {sssc }}$ in series with the line, which can control the power flow within desired values. The product of the transmission line current $\mathrm{I}_{\mathrm{m}}$ and the series voltage source $\mathrm{V}_{\text {sssc }}$, determines the active and reactive power exchanged between the series converter and the ac system. The series converter generates the reactive power exchanged at the ac terminal internally.

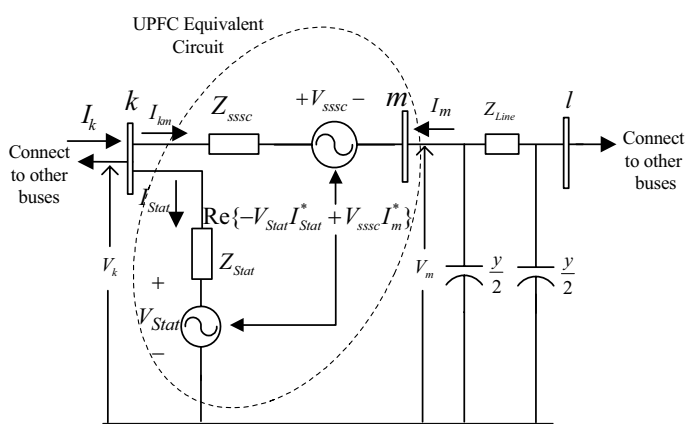

Fig 1. UPFC equivalent circuit on transmission line 


\section{MODIFICATION OF FAST DECOUPLED ALGORITHM}

In FDM load flow method, the two matrices equations to be solved iteratively are:

$[\Delta P / V]=\left[B^{\prime}\right][\Delta \theta]$
$[\Delta Q / V]=\left[B^{\prime \prime}\right][\Delta V]$

where,

$\Delta P, \Delta Q:$ active and reactive power mismatch vectors

$\Delta V, \Delta \theta$ :voltage magnitude and angle correction vectors

$B_{i k}^{\prime}=1 / X_{i k} \quad$ and $\quad B_{i k}^{\prime \prime}=X_{i k} /\left(R_{i k}^{2}+X_{i k}^{2}\right)$

$B_{i i}^{\prime}=-\sum_{i \in k} 1 / X_{i k} \quad$ and $\quad B_{i i}^{\prime \prime}=-\sum_{i \in k} B_{i k}^{\prime \prime}+S_{i k} / 2$

In order to include UPFC into the FDM, the following modifications are necessary;

The $[\mathrm{k}, \mathrm{k}]$ element of matrix B" is included with the impedance of UPFC on the shunt converter side as follow:

$B_{k k}^{\prime \prime \prime}=B_{k k}^{\prime \prime}-X_{\text {Stat }} /\left(R_{\text {Stat }}^{2}+X_{\text {Stat }}^{2}\right)$

where $R_{i k}, x_{i k}$ are resistance and reactance of branch i-k respectively and $S_{i}$ is susceptance of branch i-k. If the voltage magnitude of bus $k$ required to be controlled, bus $\mathrm{k}$ is set as $\mathrm{V}$-control type. This type of bus is defined for a bus with a fixed voltage magnitude without any generation on the bus. This definition is necessary to differentiate between buses of PV type. Equation (4) for a two-bus system of Figure 1 becomes: $\left[\begin{array}{l}\Delta Q_{k} / V_{\text {Stat }} \\ \Delta Q_{m} / V_{m}\end{array}\right]=\left[\begin{array}{cc}B_{\text {Stat }} & B_{\text {sssc }} \\ 0 & -B_{\text {sssc }}\end{array}\right]\left[\begin{array}{l}\Delta V_{\text {Stat }} \\ \Delta V_{m}\end{array}\right]$

In the other hand, if the voltage magnitude do not required to be controlled, $V_{\text {stat }}$ is replaced by $V_{k}$ and bus $\mathrm{k}$ is set as a PQ type. The matrix equation for solving UPFC parameters is as follow:

$\left[\begin{array}{lll}\frac{\partial \Delta P_{m k}}{\partial \Delta \theta_{s s s c}} & V_{s s s c} \frac{\partial \Delta P_{m k}}{\partial \Delta V_{s s s c}} & 0 \\ \frac{\partial \Delta Q_{m k}}{\partial \Delta \theta_{s s s c}} & V_{s s s c} \frac{\partial \Delta Q_{m k}}{\partial \Delta V_{s s s c}} & 0 \\ \frac{\partial \Delta P_{b b}}{\partial \Delta \theta_{s s s c}} & V_{s s s c} \frac{\partial \Delta P_{b b}}{\partial \Delta V_{s s s c}} & \frac{\partial \Delta P_{b b}}{\partial \Delta \theta_{\text {Stat }}}\end{array}\right]\left[\begin{array}{l}\Delta \theta_{s s s c} \\ \frac{\Delta V_{s s s c}}{V_{\text {sssc }}} \\ \Delta \theta_{\text {Stat }}\end{array}\right]=\left[\begin{array}{l}\Delta P_{m k} \\ \Delta Q_{m k} \\ \Delta P_{b b}\end{array}\right]$

where, $\Delta P_{m k}$ and $\Delta Q_{m k}$ are the power mismatches of power flow from bus $\mathrm{m}$ to $\mathrm{k}$ and $\Delta P_{b b}$ is the active power mismatch of UPFC source.

The above equations are solved sequentially i.e. by solving equation 5 first, then 6 and 9 or simply can be stated as [P, Q, UPFC] sequence. This process is simplified in the flow chart of Fig. 2, which shows a basic FDM algorithm that has been included with UPFC analysis. The basic algorithm is represented by solid line boxes and the new added routines are represented by dashes line boxes. The routine consists of various tasks (in the form of function) to include UPFC into the load flow analysis. For example, the routine of calculate new active mismatch will calculate the injected active power due to the shunt converter at a certain bus that connected to UPFC. This power will then be subtracted from the power mismatch that has been calculated in the previous algorithm to get new power mismatch at certain buses. By developing this routine, the original codes for calculating the active power mismatches still applicable without any modification. The same explanation also applied in recalculating new reactive power mismatches due to the injected reactive power. This explained how the UPFC steady-sate model can be integrated into FDM without changing or modifying any of the previously developed algorithm and codes.

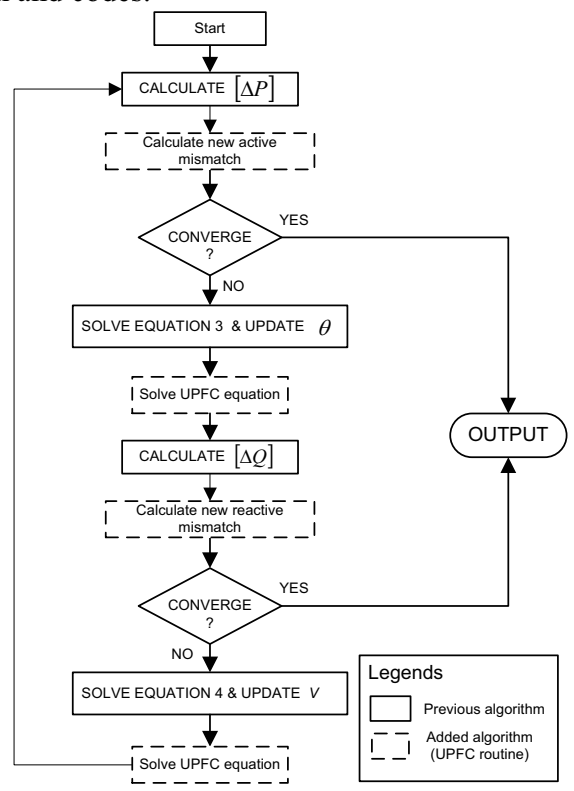

Fig 2. Fast Decoupled Load Flow Algorithm

\section{TEST CASES}

\subsection{5-Bus System Test}

The objective of this test is to validate the result obtained by suing FDM with result obtained using NRM in Ref. [5]. The same test data is used here i.e the 5-Test Bus system. The network is incorporated with UPFC between bus 3 and 4 as shown in Figure 3 together with the power flow results. The UPFC purpose on this network is to maintain active and reactive powers leaving UPFC towards bus 4 to $40 \mathrm{MW}$ and 2 MVAR respectively and also to maintain voltage magnitude on bus 3 at 1.0 p.u. The result of voltage and phase angle of the network system and UPFC sources are presented in Table 1. Whereas, the result of power flow on the network is presented in Fig. 3.

Table 1 Result of voltage magnitude and phase angle of 5- bus syst
\begin{tabular}{|l|l|l|}
\hline Bus & $\mathrm{V}$ (p.u) & $\theta(\mathrm{deg})$ \\
\hline 1 & 1.06 & 0.0 \\
\hline 2 & 1.0000 & -1.7690 \\
\hline 3 & 1.0000 & -6.0153 \\
\hline 4 & 0.9917 & -3.1903 \\
\hline 5 & 0.9745 & -4.9735 \\
\hline Auxiliary bus (6) & 0.9965 & -2.5120 \\
\hline Series source & 0.10126 & -92.7192 \\
\hline Shunt source & 1.01734 & -6.00469 \\
\hline
\end{tabular}


The power flows result shows in Figure 3 have justified the capability of FDM in solving load flow considering UPFC in the network. This result can be compared with ref. [5], and one will find the same results. All the obtained values are fulfilling the specific control requirements of power flow and voltage magnitude as well. These can be proven by calculating the power flow between bus 6 and 4 by using the obtained voltage and phase angle of related bus bars. In terms of iteration number, the FDM requires more iteration number compared to NRM.

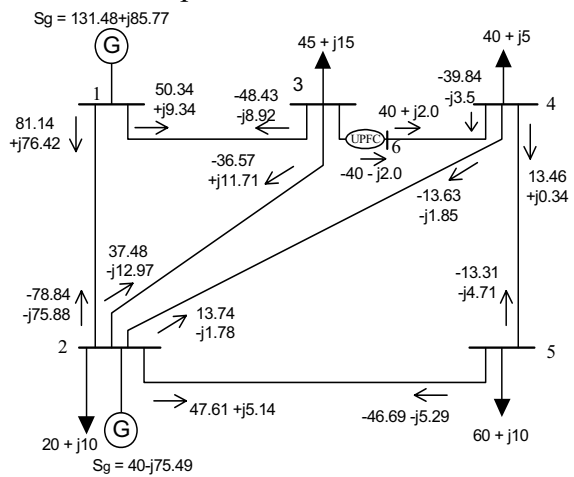

Fig 3. 5 Bus system with UPFC and load flow results

The power flows result shows in Figure 3 have justified the capability of FDM in solving load flow considering UPFC in the network. This result can be compared with ref. [5], and one will find the same results. All the obtained values are fulfilling the specific control requirements of power flow and voltage magnitude as well. These can be proven by calculating the power flow between bus 6 and 4 by using the obtained voltage and phase angle of related bus bars. In terms of iteration number, the FDM requires more iteration number compared to NRM.

\subsection{IEEE 300-Bus System Test}

The placing of UPFCs on this system is chosen randomly with the restriction that the UPFC is placed between buses that not being any subject of control. This is to avoid multiple control of bus voltage magnitude, which will only cause divergence. The position of UPFC is as shown in Fig. 1 with UPFC placed between bus $k$ and $l$. Bus $m$ is the fictitious bus and bus $k$ is the shunt side of the UPFC. The values of power flows can be controlled within specific values in any direction between bus $k$ and $l$. The voltage magnitude on bus $k$ can also be controlled so as it can be maintained to a specific value. Three test cases with particular control requirements are presented in Table 2 .

\begin{tabular}{|c|c|c|c|c|c|c|c|}
\hline \multirow{3}{*}{$\begin{array}{l}\mathscr{z} \\
\tilde{z} \\
\tilde{J}\end{array}$} & \multicolumn{2}{|c|}{ Branch } & \multicolumn{5}{|c|}{ Control Requirement } \\
\hline & \multirow[b]{2}{*}{$\overline{\underline{n}}$} & \multirow[b]{2}{*}{ 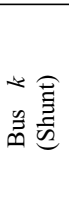 } & \multirow[b]{2}{*}{$\sum_{\substack{0 \\
<}}^{\S}$} & \multirow[b]{2}{*}{ 总 } & \multirow[b]{2}{*}{$\begin{array}{l}\stackrel{8}{\infty} \\
\stackrel{\Xi}{0} \\
\dot{0}\end{array}$} & \multicolumn{2}{|c|}{$\begin{array}{l}\text { Power flow } \\
\text { into bus }\end{array}$} \\
\hline & & & & & & 总 & 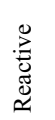 \\
\hline I & 6 & 2 & 40 & 2.0 & 1.02 & 2 & 2 \\
\hline II & 2 & 6 & 40 & No & 1.02 & 6 & No \\
\hline \multirow{3}{*}{ III } & 6 & 2 & 40 & 2.0 & 1.02 & 2 & 2 \\
\hline & 75 & 31 & 60 & 6.0 & 1.0 & 75 & 75 \\
\hline & 183 & 184 & 30 & 5 & 1.0 & 183 & 183 \\
\hline
\end{tabular}

The control specifications are indicated in the column 'control requirement'. The ability of FDM to solve multiple UPFC in the system is shown by case III. For these tests, the accuracy of convergence tolerance for power mismatch and UPFC control mismatch is set to $10^{-4}$.

The analysis results are presented in Table 3. From the results, it can be observed that although both methods produce different results, the difference is not too large. The values are satisfied the power flow and voltage control requirement, which could be justified by calculating the power flows between two buses. The convergence characteristic of load flow analysis also justified that the values obtained are correct solution. Case II shows divergence for NRM and not for FDM. This occurred in NRM because large increment in the variables value correction during the back substitution process produces large residual terms, resulting in poor convergence or may diverge since the new value is far from the right one. On the other hand, this did not

Table 3 Results test of 300 bus system

\begin{tabular}{|c|c|c|c|c|c|c|c|c|c|c|c|c|}
\hline \multirow{3}{*}{ 总 } & \multirow{3}{*}{ 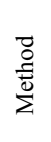 } & \multirow{3}{*}{ 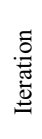 } & \multicolumn{4}{|c|}{ UPFC parameters } & \multirow{2}{*}{\multicolumn{2}{|c|}{$\begin{array}{c}\text { Bus } k \\
\text { (Shunt side) }\end{array}$}} & \multirow{2}{*}{\multicolumn{2}{|c|}{ Bus $l$}} & \multirow{2}{*}{\multicolumn{2}{|c|}{$\begin{array}{c}\text { Bus } m \\
\text { (Auxiliary bus) }\end{array}$}} \\
\hline & & & \multicolumn{2}{|c|}{ Shunt source } & \multicolumn{2}{|c|}{ Series source } & & & & & & \\
\hline & & & V & $\theta^{\circ}$ & $\mathrm{V}$ & $\theta^{\circ}$ & $\mathrm{V}$ & $\theta^{\circ}$ & $\mathrm{V}$ & $\theta^{\circ}$ & $\mathrm{V}$ & $\theta^{\circ}$ \\
\hline \multirow{2}{*}{ I } & NR & 7 & 0.84412 & 10.31888 & 0.19998 & 89.13210 & 1.02 & 10.3444 & 0.9967 & 1.5219 & 0.9966 & 1.3146 \\
\hline & FD & 8 & 0.83931 & 10.30100 & 0.20114 & 86.84460 & 1.02 & 10.3158 & 0.9944 & 1.4823 & 0.9938 & 1.2756 \\
\hline \multirow{2}{*}{ II } & NR & \multicolumn{11}{|c|}{ Diverge - UPFC limit is violated } \\
\hline & FD & 7 & 0.85626 & 9.214727 & 0.05584 & 85.28808 & 1.02 & 9.2155 & 1.0 & 3.6594 & 1.0010 & 3.8623 \\
\hline \multirow{6}{*}{ III } & \multirow{3}{*}{ NR } & \multirow{3}{*}{7} & 0.84450 & 11.09799 & 0.20082 & 89.72434 & 1.02 & 11.1247 & 0.9964 & 2.2582 & 0.9963 & 2.0507 \\
\hline & & & 0.94554 & -4.36040 & 0.26497 & 75.14068 & 1.0 & -4.4174 & 1.0166 & -11.8109 & 0.9856 & -16.1862 \\
\hline & & & 1.05089 & -30.26503 & 0.03909 & 18.32633 & 1.0 & -30.201 & 0.9844 & -28.7883 & 0.9716 & -30.0911 \\
\hline & \multirow{3}{*}{ FD } & \multirow{3}{*}{16} & 0.83996 & 11.05718 & 0.20201 & 87.53756 & 1.02 & 11.0721 & 0.9942 & 2.19095 & 0.9936 & 1.98335 \\
\hline & & & 0.94087 & -4.54728 & 0.25937 & 72.15076 & 1.0 & -4.6135 & 1.0156 & -11.8264 & 0.9782 & -16.026 \\
\hline & & & 1.04401 & -30.27939 & 0.05861 & -5.58871 & 1.0 & -30.1938 & 0.9702 & -28.6232 & 0.9518 & -29.7632 \\
\hline
\end{tabular}


occurred in FDM since the correction values are usually very small, which makes convergence process slow and therefore the FDM has high possibility to converge. To solve divergence problem in NRM, the correction value is required to be limited during the backward substitution as proposed in [4]. However, this still doesn't guarantee $100 \%$ convergence in NRM since the limitation value is chosen randomly. Therefore, the FDM could be used for cases where NRM analysis produces divergence. The result of one or two iteration from FDM also can be used as the initial value for NRM, which normally can make the NRM solution to converge.

\section{CONVERGENCE CHARACTERISTIC}

Another important characteristic that need to be investigated is on the convergence characteristics when UPFC presents in the network. In order observed this characteristic, graphs on the power mismatches as a function of number of iteration are drawn. The following figures are graphs correspond to IEEE 300-bus test system without UPFC and with UPFC for case III.

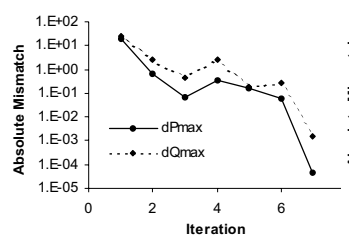

(a)

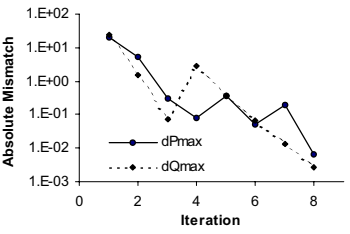

(b)
Fig 4. Absolute power mismatches graphs for 300 Bus system without UPFC (a) NRM (b) FDM

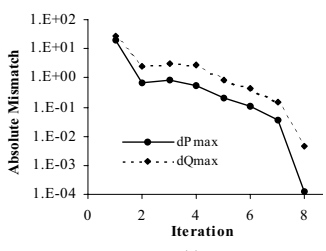

(a)

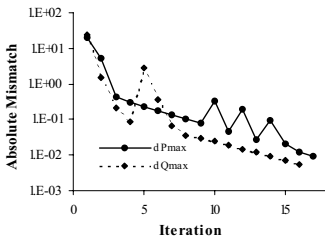

(b)
Fig 5. Absolute power mismatches graphs for 300 Bus system with UPFC for case III (a) NRM (b) FDM

There is common characteristic in the above FDM graphs, where the absolute active power mismatches oscillate before it converging. From observation it was found that the bus where oscillation occurs is connected to either a variable tap transformer or phase shifter, and not connected to UPFC. This means that the UPFC control mismatches are not the cause of oscillation. It also can be observed from the above graphs that both methods produced similar convergence characteristic as without UPFC, however with extra iteration numbers.

For NRM, the number of iteration does not increase significantly although to the system containing UPFC. On the other hand, the number of iteration for FDM increased significantly when UPFC exists in the system. This occurred since the FDM was using sequential approach, where the basic load flow equation is solve separately from the UPFC equations, resulting in unequal convergence time of the power mismatches and UPFC mismatches control. Therefore, FDM requires additional iteration numbers before converging compared to NRM. Whereas in NRM, a unified approach was applied that made the UPFC equations being solved in the same time with the basic load flow variables in the same matrix equation. Therefore, the powers and UPFC control mismatches converged at the same time.

\section{CONCLUSION}

This paper has discussed the modification of the basic FDM algorithm to consider UPFC in the analysis. A sequential approach has been used, which made the UPFC equations separated from the basic FDM equations. By using this approach, the integration of the UPFC model into the FDM is done without changing or modifying any of the basic FDM algorithms. A basic FDM source codes that has been developed therefore can be added with this model without affecting the existing codes.

The results test of FDM shows the ability of FDM to solve networks containing UPFC device. Although most of the cases, FDM consumed more iterations number than NRM, convergence still can be meet and the obtained results fulfilled the control requirements. Comparison with NRM results also shows similar results, where the values are not too large. It can be concluded that the steady-state UPFC model that was used for NRM also suitable to be used for FDM. This proposed FDM algorithm can be used as the alternative method to solve problems, where NRM cannot work. It also can be used to get initial values before applying NRM.

\section{ACKNOWLEDGMENTS}

The authors gratefully acknowledge the assistance rendered by the Department of Electrical Engineering and the Faculty of Engineering, University of Malaya in the work reported in this paper.

\section{REFERENCES}

[1] Narain G.Hingorani and Laszlo Gyugyi, "Understanding FACTS Concept and Technology of Flexible AC Transmission System", IEEE Press, 2000.

[2] Noroozian, M., and etc, "Use of UPFC for Optimal Power Flow Control", IEEE/KTH Stockholm Power tech. Conference, pp 506-511, 1999.

[3] Nabavi-Niaki and M.R. Iravani, "Steady state and Dynamic Models of Unified Power Flow Controller (UPFC) for Power System Studies", IEEE Trans On Power System, Vol.11, No 4, pp 1937-1943, Nov 1996.

[4] C.R. Fuerter-Esquivel, E. Acha and H. Ambriz-Perez, "A Comprehensive Newton-Raphson UPFC Model for the Quadratic Power Flow Solution of Practical Power Networks", IEEE Trans. on Power System, Vol. 15, No 1, pp 102-109, Feb. 2000.

[5] C.R. Fuerter-Esquivel, E. Acha, "Unified Power Flow Controller: A Critical Comparison of Newton-Raphson UPFC Algorithm in Power Flow Studies", IEE Proc. Gener. Transm. Distrib. , Vol. 144, No 5, pp. 437-44, Sept. 1997. 